Bull. Austral. Math. Soc.

Vol. 52 (1995) [91-96]

\title{
SETS OF DIFFERENTIALS AND SMOOTHNESS OF CONVEX FUNCTIONS
}

\author{
WeE-KeE Tang
}

\begin{abstract}
Approximation by smooth convex functions and questions on the Smooth Variational Principle for a given convex function $f$ on a Banach space are studied in connection with majorising $f$ by $C^{1}$-smooth functions.
\end{abstract}

It is known that a weakly compactly generated (WCG) Banach space admits an equivalent Fréchet differentiable norm if it admits a Fréchet differentiable bump function (see, for example [5]). However, there are nonseparable spaces that admit Fréchet differentiable bump functions but admit no equivalent Fréchet differentiable norm (see, for example [3, Chapter VII]). If the space $X$ admits an equivalent norm with modulus of smoothness of power type 2, then every convex continuous function on $X$ has points of Lipschitz smoothness (see, for example [3, Chapter IV]). The purpose of this note is to localise these results. We prove that any convex Lipschitz function $f$ that is defined on a WCG Banach space $X$ can be uniformly approximated by Fréchet differentiable convex functions if $f$ is majorised on $X$ by a Fréchet smooth convex function. If, moreover, $\overline{s p a n}^{\|} \|\{\partial f(x): x \in X\}$ is a subspace of $X^{*}$ that admits a norm with modulus of rotundity of power type 2 , then there is a convex function $\psi$ with $\psi^{\prime}$ Lipschitz on $X$ such that $\psi \geqslant f$ on $X$ and $\psi(x)=f(x)$ for some $x \in X$. Thus in particular, $f$ has points of Lipschitz smoothness. A separable version of these problems was studied in [10]. We use standard notation in this note (see for example [3]), and refer to $[6,7,9]$ and [3] for some unexplained notions and results used in this note.

THEOREM 1. Let $f$ be a convex Lipschitz function defined on a WCG Banach space $X$. Then the following are equivalent.

(1) The function $f$ can be uniformly approximated on $X$ by a Fréchet differentiable convex function.

(2) There exists a Fréchet differentiable convex function $\phi$ defined on $X$ such that $\phi \geqslant f$ on $X$.

\section{Received 15th October, 1994}

The author was supported by NSERC (Canada). The author is indebted to Professor V. Zizler for his guidance and suggestions, and to the referee for his/her helpful comments.

Copyright Clearance Centre, Inc. Serial-fee code: 0004-9729/95 \$A2.00+0.00. 
Proof: Clearly (1) $\Longrightarrow(2)$. The proof $(2) \Longrightarrow(1)$ is divided into a few steps.

Proposition 2. Let $X$ be a WCG Banach space, $\phi$ be a Fréchet differentiable convex function defined on $X$ and let $Y:=\overline{s p a n}\|\cdot\|\left\{\phi^{\prime}(x): x \in X\right\}$. Then there exists a projectional resolution of the identity (PRI) $\left\{P_{\alpha}: \omega \leqslant \alpha \leqslant \mu\right\}$ on $X$ such that

(i) $P_{\mu}^{*}=I,\left\|P_{\alpha}^{*}\right\|=1$ for all $\alpha$.

(ii) $P_{\alpha}^{*} P_{\beta}^{*}=P_{\beta}^{*} P_{\alpha}^{*}=P_{\min (\alpha, \beta)}^{*}$.

(iii) $P_{\alpha}^{*} Y \subset Y$ for all $\alpha$.

(iv) $\operatorname{dens}\left(P_{\alpha}^{*} Y\right) \leqslant \alpha$ for all $\alpha \leqslant \mu$.

(v) $P_{\alpha}^{*} Y=\overline{s p a n}\|\cdot\| \bigcup_{\beta<\alpha} P_{\beta+1}^{*} Y$ for all $\alpha \leqslant \mu$.

PROOF: Using standard techniques for constructing projectional resolutions of the identity (see for example [3, Chapter VI]), we only need to show $P_{\alpha}^{*} Y \subset Y$ and $P_{\alpha}^{*} Y=$ $\overline{\operatorname{span}^{\|} \|} \bigcup_{\beta<\alpha} P_{\beta+1}^{*} Y$ for all $\alpha$. The proof of this is contained in Lemmas 3 to 5 .

LEMma 3. In notation as above, we can construct a $P R I\left\{P_{\alpha}: \omega \leqslant \alpha \leqslant \mu\right\}$ so that $P_{\alpha}^{*} \phi^{\prime}(y)=\phi^{\prime}(y)$ for all $y \in P_{\alpha} X$.

Proof: See Lemma 5 in [5].

Lemma 4. With notation as above, $P_{\alpha}^{*} Y=\overline{\operatorname{span}}\|\cdot\|\left\{\phi^{\prime}(x): x \in P_{\alpha} X\right\}$.

Proof: To see $P_{\alpha}^{*} Y \supset \overline{\operatorname{span}\|\cdot\|}\left\{\phi^{\prime}(x): x \in P_{\alpha} X\right\}$, we let $x \in P_{\alpha} X$, and show that $\phi^{\prime}(x) \in P_{\alpha}^{*} Y$. Since $\phi$ is $C^{1}$-smooth, given $\varepsilon>0$, there exists an $x_{\beta} \in P_{\beta+1} X$ for some $\beta<\alpha$, such that $\left\|\phi^{\prime}(x)-\phi^{\prime}\left(x_{\beta}\right)\right\|<\varepsilon$. By Lemma 3, $\phi^{\prime}\left(x_{\beta}\right)=P_{\beta+1}^{*} \phi^{\prime}\left(x_{\beta}\right)$. Therefore $\phi^{\prime}\left(x_{\beta}\right) \in P_{\beta+1}^{*} Y \subset P_{\alpha}^{*} Y$. As $P_{\alpha}^{*} Y$ is closed, $\phi^{\prime}(x) \in P_{\alpha}^{*} Y$. For the converse inclusion, we follow the idea in [4]. Let $\phi^{\prime}(x) \in Y$. Clearly $g(\cdot)=\phi(\cdot)-\phi^{\prime}(x)(\cdot)$ is a continuous bounded below function on $X$. Hence its restriction $g_{\mid P_{\alpha} X}$ is also continuous and bounded below. By Ekeland's variational principle, given $\varepsilon>0$, there exists $x_{\alpha} \in P_{\alpha} X$ such that for every $w \in B_{P_{\alpha} X}, t>0$, we have $g\left(x_{\alpha}+t w\right) \geqslant$ $g\left(x_{\alpha}\right)-\varepsilon t$, thus, $\phi^{\prime}(x)(w) \leqslant\left(\phi\left(x_{\alpha}+t w\right)-\phi\left(x_{\alpha}\right)\right) / t-\varepsilon$. Hence, by taking limits, we have $\phi^{\prime}(x)(w)-\phi^{\prime}\left(x_{\alpha}\right)(w) \leqslant \varepsilon$. Therefore $\sup \left\{\left|\phi^{\prime}(x)(v)-\phi^{\prime}\left(x_{\alpha}\right)(v)\right|: v \in B_{P_{\alpha} X}\right\} \leqslant$ $\varepsilon$. Given any $h \in B_{X}$, we have $\left(h, P_{\alpha}^{*} \phi^{\prime}(x)-\phi^{\prime}\left(x_{\alpha}\right)\right)=\left(h, P_{\alpha}^{*} \phi^{\prime}(x)-P_{\alpha}^{*} \phi^{\prime}\left(x_{\alpha}\right)\right)=$ $\left(P_{\alpha} h, \phi^{\prime}(x)-\phi^{\prime}\left(x_{\alpha}\right)\right) \leqslant \varepsilon$. Therefore $\left\|P_{\alpha}^{*} \phi^{\prime}(x)-\phi^{\prime}\left(x_{\alpha}\right)\right\| \leqslant \varepsilon$. Finally, since $Y$ is the closed linear span of the derivatives of $\phi$ and $P_{\alpha}$ is bounded, the assertion follows.

LEMma 5. $P_{\alpha}^{*} Y=\overline{\text { span }}\|\cdot\| \bigcup_{\beta<\alpha} P_{\beta+1}^{*} Y$ for every $\alpha \leqslant \mu$.

Proof: Clearly $P_{\alpha}^{*} Y \supset \overline{s p a n}\|\cdot\| \bigcup_{\beta<\alpha} P_{\beta+1}^{*} Y$. The converse inclusion follows from Lemma 4 and the continuity of $\phi^{\prime}$.

Proof of Theorem 1: Since $f \leqslant \phi$, using Ekeland's variational principle as in Lemma 4 we show that $\operatorname{dom} f^{*} \subset Y$. Using Lemma 5, and the classical Troyanski's 
construction (see for example [3, Chapter VII]) we obtain a dual norm $\|\cdot\|^{*}$ in $X^{*}$ such that its restriction on $Y$ is locally uniformly rotund (LUR). Define a sequence of functions $\left\{h_{n}\right\}$ on $X^{*}$ by $h_{n}\left(x^{*}\right)=f^{*}\left(x^{*}\right)+\left\|x^{*}\right\|^{* 2} /\left(4 n^{4}\right)$. Clearly, dom $h_{n}=\operatorname{dom} f^{*}$. Define $g_{n}:=f \square n^{4}\|\cdot\|^{2}$, where $\square$ denotes the infimal convolution. Note that $g_{n}$ is convex and continuous on $X$ and $g_{n}^{*}=h_{n}$ for all $n$. Given $n \in \mathbb{N}, x \in X$ and $y \in \partial g_{n}(x)$, note that $h_{n}$ is rotund at $y$ with respect to $x$ in the sense of [1], that is, for every $\varepsilon>0$, there exist $\delta>0$ such that $\left\{v: h_{n}(y+v)-h_{n}(y)-(x, v) \leqslant \delta\right\} \subset \varepsilon B_{X}$. (see, $[10])$. By [1, Proposition 4], $g_{n}$ is Fréchet differentiable at $x$ with the derivative $y$. One can also show that $\lim g_{n}=f$ uniformly on X (see for example [8, Lemma 2.4]).

Since the function $f$ can be quite "flat" in Theorem 1, there is a difficulty in applying the techniques of Smooth Variational Principles (see, [3, Chapter I]) in this situation. However, under more restrictive assumptions we can use the Stegall-Fabian variational principle and obtain our variational result by duality. We shall say that $x \in X$ is a point of Lipschitz smoothness of a convex function $f$ if $f(x+h)+f(x-h)-$ $2 f(x)=O\left(\|h\|^{2}\right)$.

Lemma 6. Let $f$ be a convex continuous function on a Banach Space $X$ and $g$ be its dual function. Suppose there exists a constant $C$ such that for any $x \in X$, $y \in \partial f(x)$, and for any $\varepsilon>0$, we have

$$
\left\{v: g(y+v)-g(y)-(x, v) \leqslant C \varepsilon^{2}\right\} \subset \varepsilon B_{X^{*}} .
$$

Then $f$ is Fréchet differentiable and $f^{\prime}$ is Lipschitz on $X$.

Proof: By taking polars, we have $\varepsilon^{-1} B_{X} \subset\left\{v: g(y+v)-g(y)-(x, v) \leqslant C \varepsilon^{2}\right\}^{0}$. According to Proposition 3 of $[1],\left\{v: g(y+v)-g(y)-(x, v) \leqslant C \varepsilon^{2}\right\}^{0} \subset C^{-1} \varepsilon^{-2}\{u$ : $\left.f(x+u)-f(x)-(y, u) \leqslant C \varepsilon^{2}\right\}$. Therefore, $\varepsilon C B_{X} \subset\{u: f(x+u)-f(x)-(y, u) \leqslant$ $\left.C \varepsilon^{2}\right\}$, that is, for any $u \in \varepsilon C B_{X}, f(x+u)+f(x-u)-2 f(x) \leqslant 2 / C(\varepsilon C)^{2}$. Thus $f^{\prime}$ exists at $x$ and we have that $f^{\prime}$ is Lipschitz on $X$ (see, for example [3, Lemma V.3.5]).

THEOREM 7. Let $f$ be a Lipschitz convex function on a Banach space $X$ and $Y=\overline{\text { span }}^{\|\cdot\|}\{\partial f(x): x \in X\}$. Suppose that $Y$ admits an equivalent norm with modulus of convexity of power type 2 . Then $f$ can be majorised by a convex function $\psi$ that has a Lipschitz derivative and $\psi(x)=f(x)$ for some $x \in X$. In particular, $f$ has points of Lipschitz smoothness.

Proof: Let $\|\cdot\|$ be an equivalent norm on $X^{*}$ such that its restriction on $Y$ has modulus of convexity of power type 2 (see, for example [3, Lemma II.8.1]). We note that $Y$ is $w^{*}$-closed. Indeed, since $Y$ is reflexive, $B_{Y}$ is compact in the weak topology 
of $X^{*}$ and thus $B_{Y}$ is $w^{*}$-compact in $X^{*}$. By the Banach-Dieudonne theorem, $Y$ is $w^{*}$-closed. Assume that $f(0)=0$, and thus we have $f^{*} \geqslant 0$ on $X^{*}$. Let

$$
h\left(x^{*}\right)=\left\{\begin{array}{l}
\frac{1}{2}\left\|x^{*}\right\|^{2}-\frac{1}{2} m^{2} \text { if } x^{*} \in Y \\
\infty \text { otherwise }
\end{array}\right.
$$

where $m=\operatorname{Lip}(f)$. Since $Y$ is $w^{*}$-closed, $h$ is $w^{*}$-lower semicontinuous and $h=$ $\left(h_{\mid X}^{*}\right)^{*}$. We show that $h$ satisfies the condition on the function $g$ given in Lemma 6 . Indeed, by the modulus of rotundity of $\|\cdot\|$, there exists $L>0$ such that for any $y_{1}, y_{2} \in Y$, we have

$$
\frac{1}{2}\left\{\left\|y_{1}\right\|^{2}+\left\|y_{2}\right\|^{2}\right\}-\left\|\frac{y_{1}+y_{2}}{2}\right\|^{2} \geqslant L\left\|y_{1}-y_{2}\right\|^{2}
$$

(see, for example [2, Lemma 5.I.4]). Assume that for every $k \in \mathbb{N}$ there exist $\varepsilon_{k}>0$, $x_{k} \in X, y_{k} \in \partial h_{\mid X}^{*}\left(x_{k}\right)$ and $v_{k} \in X^{*},\left\|v_{k}\right\|>\varepsilon_{k}$, such that $h\left(y_{k}+v_{k}\right)-h\left(y_{k}\right)-$ $v_{k}\left(x_{k}\right) \leqslant \varepsilon_{k}^{2} / k$. Then $\left\|y_{k}+v_{k}\right\|^{2} / 2-\left\|y_{k}\right\|^{2} / 2-\left(x_{k}, v_{k}\right) \leqslant \varepsilon_{k}^{2} / k$ for all $k$. From the definition of a subdifferential, we have $-\left(x_{k}, v_{k}\right) \geqslant\left\|y_{k}\right\|^{2}-\left\|y_{k}+v_{k} / 2\right\|^{2}$. Therefore, $\left(\left\|y_{k}\right\|^{2}+\left\|y_{k}+v_{k}\right\|^{2}\right) / 2-\left\|y_{k}+v_{k} / 2\right\|^{2} \leqslant \varepsilon_{k}^{2} / k \leqslant\left\|v_{k}\right\|^{2} / k$, which contradicts (*). Now, for each $x^{*} \in \operatorname{dom} f^{*} \subset m B_{X^{*}}$, we have $h\left(x^{*}\right) \leqslant 0 \leqslant f\left(x^{*}\right)$. Therefore $f^{*}-h$ is a lower semicontinuous convex function on $\operatorname{dom} f^{*}$ that is bounded below. Note that $f^{*}-h \geqslant\|\cdot\|-m$. By the Stegall-Fabian result (see, for example [9, Corollary 5.22]), there exists $\widehat{x} \in Y^{*}$ such that $f^{*}-h-\widehat{x}$ attains its minimum in $\operatorname{dom} f^{*}$, that is, there is a $x^{*} \in \operatorname{dom} f^{*}$ such that $f^{*}\left(x^{*}\right)-h\left(x^{*}\right)-\widehat{x}\left(x^{*}\right)=\alpha \leqslant f^{*}\left(y^{*}\right)-h\left(y^{*}\right)-\widehat{x}\left(y^{*}\right)$ for all $y^{*} \in \operatorname{dom} f^{*}$. Therefore we have $h(\cdot)+\widehat{x}(\cdot)+\alpha \leqslant f^{*}(\cdot)$ on $\operatorname{dom} f^{*}$ and the equality holds at $x^{*}$. Since $Y$ is reflexive, there exists $x \in X$ such that $y^{*}(x)=\widehat{x}\left(y^{*}\right)$ for each $y^{*} \in Y$. Let $k: X^{*} \rightarrow \mathbb{R}$ be a function defined by $k(\cdot)=h(\cdot)+x(\cdot)+\alpha$. Then $k$ is a convex function such that $k \leqslant f^{*}$ and $k\left(x^{*}\right)=f^{*}\left(x^{*}\right)$. Put $l=k_{\mid Y}$. The function $l$ is continuous and convex on $Y$. Let $\widehat{y} \in \partial l\left(x^{*}\right) \subset Y^{*}$. As $Y$ is reflexive, there exists $y \in X$ such that $\widehat{y}\left(y^{*}\right)=y^{*}(y)$ for each $y^{*} \in Y$. We claim that $y \in \partial k\left(x^{*}\right)$. Indeed, let $z^{*} \in X^{*}$. If $z^{*} \in Y, y\left(z^{*}-x^{*}\right)=\widehat{y}\left(z^{*}-x^{*}\right) \leqslant k\left(z^{*}\right)-k\left(x^{*}\right)$. If $z^{*} \notin Y$, then $y\left(z^{*}-x^{*}\right)<k\left(z^{*}\right)-k\left(x^{*}\right)=\infty$. Hence $y \in \partial k\left(x^{*}\right)$. Since $k\left(x^{*}\right)=f^{*}\left(x^{*}\right)$, we have $y \in \partial f^{*}\left(x^{*}\right)$. Thus $k^{*}(y)+k\left(x^{*}\right)=\left(x^{*}, y\right)=f^{*}\left(x^{*}\right)+f(y)$. Therefore $f(y)=k^{*}(y)$. Since $f^{*} \geqslant k$, we have $k^{*} \geqslant f$. Put $\psi=k_{\mid X}^{*}$. The function $\psi$ has a Lipschitz derivative and is our required function. Indeed, $k^{*}=(h(\cdot)+x(\cdot)+\alpha)^{*}=$ $(h+x)^{*}-\alpha=h^{*}(\cdot) \square \delta_{x}(\cdot)-\alpha=h^{*}(\cdot-x)-\alpha$ (where $\delta_{x}$ is the indicator function of the singleton $\{x\})$ and $h^{*}$ has the desired differentiability by Lemma 6 . Finally, since $f(y)=k^{*}(y)=\psi(y)$ and $f \leqslant \psi$ on $X$, we have $f(y+v)+f(y-v)-2 f(y) \leqslant$ $\psi(y+v)+\psi(y-v)-2 \psi(y) \leqslant C\|v\|^{2}$, for some constant $C$. Therefore the function $f$ is Fréchet differentiable at $y$ and $f^{\prime}$ is Lipschitz at $y$. 
Similarly, using Troyanski's result that reflexive spaces admit equivalent LUR norms (see, for example [3, Chapter VII]], we can show the following result.

Corollary 8. Let $f$ be a Lipschitz convex function on a Banach space $X$ and $Y=\overline{\text { span }}\|\cdot\| \partial f(x): x \in X\}$. If $Y$ is reflexive, then $f$ can be majorised on $X$ by a convex function $\phi$ that is Fréchet differentiable and $\phi(x)=f(x)$ for some $x \in X$.

Under the assumptions in Theorem 7, the techniques in Theorem 1 may be applied to obtain approximation by functions with Lipschitz derivatives.

Theorem 9. Let $X, Y$ and $f$ be as in Theorem 7. Then $f$ can be uniformly approximated on $X$ by convex functions that have a Lipschitz derivative.

Proof: As in the proof of Theorem 7, let $\|\cdot\|$ be an equivalent norm of $X^{*}$ such that its restriction on $Y$ is LUR. Let $h=\|\cdot\|^{2} / 2$ and $g:=h+f^{*}$ on $X^{*}$. The function $g$ is $w^{*}$-lower semicontinuous on $X^{*}$. Let $k$ be a convex function on $X$ such that $k^{*}=g$. We claim that there exists a constant $C$ such that for any $\varepsilon>0$, $x \in X$ and $y \in \partial k(x)$, we have $\{v: g(v+y)-g(y)-(x, v) \leqslant C \varepsilon\} \subset \varepsilon B_{X^{*}}$. Since $g(u)=\infty$ whenever $u \notin Y$, we only need to consider points in $Y$. Let $v \in Y$, then $(g(y)+g(y+v)) / 2-g((2 y+v) / 2) \geqslant(h(y)+h(y+v)) / 2-h((2 y+v) / 2)$ for any $y \in Y$. Using $\left({ }^{*}\right)$, we have $(g(y)+g(y+v)) / 2-g((2 y+v) / 2) \geqslant L\|v\|^{2}$ for any $v \in Y$ and for any $y \in Y$. Following the same idea as in the proof of Theorem 7, we can complete the proof of the claim. By Lemma $6, k$ is Fréchet differentiable and $k^{\prime}$ is Lipschitz. For each $n \in \mathbb{N}$ define $g_{n}:=f^{*}+h /\left(2 n^{4}\right)$ and $k_{n}$ such that $k_{n}^{*}=g$. By the above argument, the function $k_{n}$ is Fréchet differentiable and $k_{n}^{\prime}$ is Lipschitz for each $n \in \mathbb{N}$. By [8, Lemma 2.1], lim $g_{n}=f$ uniformly on $X$.

\section{REFERENCES}

[1] E. Asplund and R.T. Rockafellar, 'Gradients of convex functions', Trans. Amer. Math. Soc. 139 (1968), 443-467.

[2] B. Beauzamy, Introduction to Banach spaces and their geometry, Math. Stud. 68 (North Holland, 1985).

[3] R. Deville, G. Godefroy and V. Zizler, Smoothness and renormings in Banach spaces, Monographs and Surveys Pure Appl. Math. 64 (Pitman, 1993).

[4] M. Fabian, 'On Projectional resolution of identity on the duals of certain Banach spaces', Bull. Austral. Math Soc. 35 (1987), 363-371.

[5] K. John and V. Zizler, 'Smoothness and its equivalents in weakly compactly generated Banach spaces', J. Funct. Anal. 15 (1974), 161-166.

[6] J. Lindenstrauss and L. Tzafrifi, Classical Banach spaces Vol. I, Ergebnisse der Mathematik und ihrer Grenzgebiete 92 (Springer-Verlag, Berlin, Heidelberg, New York, 1977).

[7] J. Lindenstrauss and L. Tzafrif, Classical Banach spaces Vol. II, Ergebnisse der Mathematik und ihrer Grenzgebiete 97 (Springer-Verlag, Berlin, Heidelberg, New York, 1979). 
[8] D. McLaughlin, R.A. Poliquin, J.D. Vanderwerff and V.E. Zizler, 'Second order Gateâux differentiable bump functions and approximations in Banach spaces', Canad. J. Math 45 (1993), 612-625.

[9] R.R. Phelps, Convex functions, monotone operators and differentiability, Lecture Notes in Mathematics 1364, (Second Edition) (Springer-Verlag, Berlin, Heidelberg, New York, 1993).

[10] W-K. Tang, 'On Fréchet differentiability of convex functions on Banach spaces' (to appear).

Department of Mathematics

University of Alberta

Edmonton

Canada T6G 2G1 